\title{
Effect of an Arm Traction Device on Image Quality and Radiation Exposure during Neck CT: A Prospective Study
}

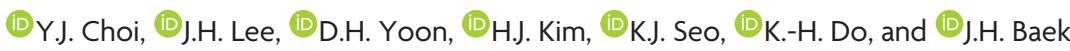

\begin{abstract}
BACKGROUND AND PURPOSE: The image quality of neck CT is frequently disturbed by streak artifact from the shoulder girdles. Our aim was to determine the effects of an arm traction device on image quality and radiation exposure in neck CT.
\end{abstract}

\begin{abstract}
MATERIALS AND METHODS: Patients with lymphoma with complete remission who were scheduled to undergo 2 consecutive follow-up neck CT scans for surveillance within a 1-year interval were enrolled in this prospective study. They underwent 2 consecutive neck CT scans (intervention protocol: patients with an arm traction device; standard protocol: no positioning optimization) on the same CT system. The primary outcome measures were image noise in the lower neck and dose-length product. Secondary outcomes were streak artifacts in the supraclavicular fossa, volume CT dose index, and the extent of the biacromial line shift.
\end{abstract}

RESULTS: Seventy-three patients were enrolled and underwent 2 consecutive CT scans with a mean interval of 155 days. In the intervention protocol, a mean noise reduction in the lower neck of $25.2 \%-28.5 \%(P<.001)$ was achieved, and a significant decrease in dose-length product (413 versus 397, $P<.001$ ) was observed. The intervention protocol significantly decreased streak artifacts $(P<.001)$ and volume CT dose index (13.9 versus 13.4, $P<.001)$ and could lower the biacromial line an average of $2.1 \mathrm{~cm}$.

CONCLUSIONS: An arm traction device can improve image quality and reduce radiation exposure during neck CT. The device can be simply applied in cooperative patients with suspected lower neck lesions, and the approach offers distinct advantages over the conventional imaging protocol.

ABBREVIATIONS: CTDI $_{\text {vol }}=$ volume $C T$ dose index; $\mathrm{DLP}=$ dose-length product

$\mathbf{T}$ he image quality of CT in the lower neck is frequently disturbed by streak artifacts from the shoulder girdles and iodinated contrast media remaining in the subclavian vein. CT image-quality improvement strategies can involve trade-offs, including factors that increase the radiation exposure or the manufacturing cost related to CT hardware or software improvement. ${ }^{1-7}$ Previous studies have tried to improve image quality by optimization of the shoulder position without the need for increased radiation exposure or the additional cost requirements of complex data processing. ${ }^{8-11}$ One such study demonstrated that an arm traction device combined with au-

Received April 24, 2017; accepted after revision August 14.

From the Departments of Radiology and Research Institute of Radiology (Y.J.C., J.H.L., K.J.S., K.-H.D., J.H.B.), Oncology (D.H.Y.), and Clinical Epidemiology and Biostatistics (H.J.K.), University of Ulsan College of Medicine, Asan Medical Center, Seoul, Korea.

Please address correspondence to Jeong Hyun Lee, MD, PhD, Department of Radiology and Research Institute of Radiology, University of Ulsan College of Medicine, Asan Medical Center, 86 Asanbyeongwon-Gil, Songpa-Gu, Seoul 138-736, Republic of Korea; e-mail: jeonghlee@amc.seoul.kr

http://dx.doi.org/10.3174/ajnr.A5418 tomatic tube current modulation reduced the image noise and streak artifacts in the lower neck, while also decreasing the radiation exposure level. ${ }^{9}$ Although the authors suggested the potential utility of an arm traction device that worked by lowering the shoulder level, the patient characteristics demonstrated considerable heterogeneity and the CT machines varied between the control and intervention groups; it is possible that these may have influenced the study results. Therefore, the purpose of this study was to prospectively determine any additive effects of an arm traction device and automatic tube current modulation on the image quality and radiation exposure of CT of the neck region. This was performed by imaging the same subjects in both the intervention protocol (using the arm traction device) and the standard protocol and performing all acquisitions on the same CT scanner.

\section{MATERIALS AND METHODS}

The prospective study protocol was reviewed and approved by the hospital review board, and the requirement for informed consent for data evaluation was waived. Written informed consent to un- 


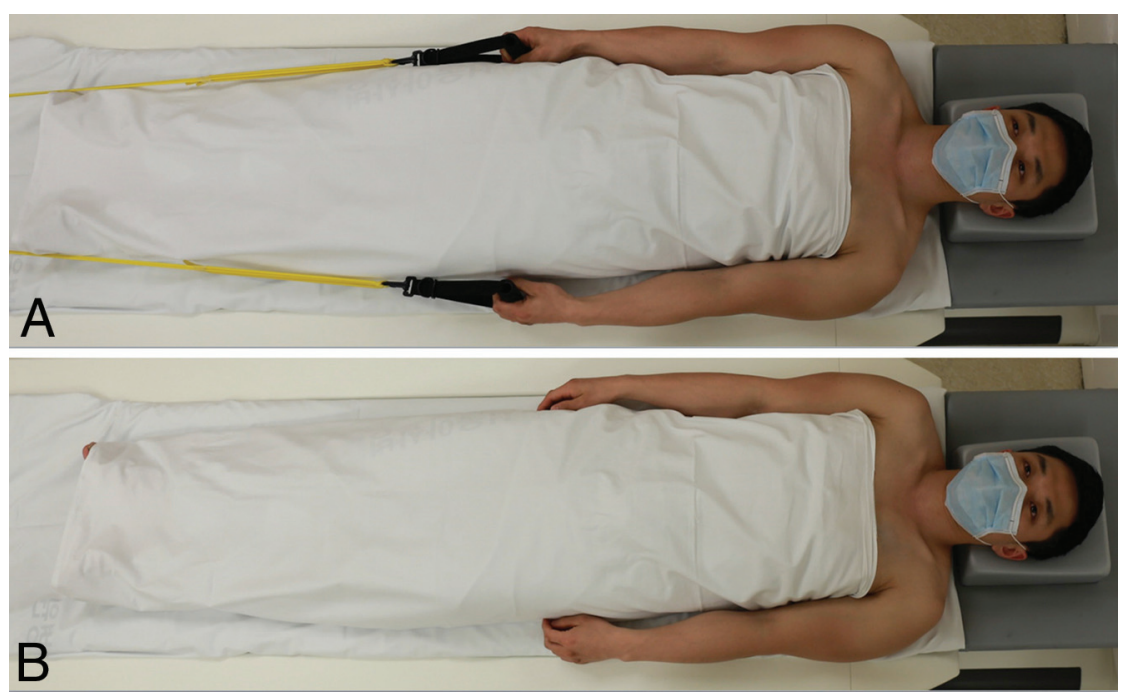

FIG 1. Patient position for neck CT imaging. Patients in the intervention $C T$ protocol were examined with the arm traction device (A). Patients in the standard CT protocol were examined in a relaxed supine position (B). Reprinted with permission from Choi et al. ${ }^{9}$

dergo the CT protocol was obtained from all patients before each examination.

\section{Study Patients}

Patients were eligible if they met the following criteria: older than 18 years of age; able to follow commands and cooperate with the use of the arm traction device; and patients with lymphoma with complete remission who were scheduled to undergo 2 consecutive follow-up neck CT scans within a 1-year interval for surveillance purposes. Complete remission of lymphoma was defined as no evidence of disease, as indicated by radiologic imaging, such as CT or $\left[{ }^{18} \mathrm{~F}\right]$ fluorodeoxyglucose positron-emission tomography. $\mathrm{Pa}-$ tients were excluded from the study if they met any of the following criteria: a previous history of surgery or radiation therapy to the neck; vascular or bone-related medical implants such as a central line or a metallic plate in the neck, upper arms, or shoulder; suspicious disease progression on follow-up CT; or an interval of $>1$ year between the 2 consecutive CT scans.

\section{Outcome Measures}

This study had 2 primary outcomes: the image quality and radiation exposure from the CT scan. CT image quality was measured by image noise at the level of the lower neck, while radiation exposure was measured by the dose-length product (DLP). Secondary outcomes were streak artifacts in the supraclavicular fossa, image noise in the midneck, volume CT dose index $\left(\mathrm{CTDI}_{\mathrm{vol}}\right)$, effective diameter, and the extent of shoulder level lowering measured by the extent of the biacromial line shift.

\section{CT Protocol and Analysis}

Patients underwent 2 consecutive neck CT scans (intervention and standard protocol). In the standard protocol, patients were positioned on the CT table with the shoulders lowered as much as possible and told to maintain the position throughout the scan. In the intervention protocol, a custom-made arm traction device was applied to the patients. This device was placed and secured by a radiologic technologist (Fig 1).

All patients underwent CT examinations on the same 128-channel multidetector CT system (Somatom Definition Edge; Siemens, Erlangen, Germany). Imaging variables were as follows: 120 kV; 200 effective mAs; axial scan mode; display FOV, $22 \mathrm{~cm}$; large-body scan FOV, $50 \mathrm{~cm}$; pitch, 1; gantry rotation time, 0.5 seconds; detector collimation, $128 \times 0.6 \mathrm{~mm}$; and $3-\mathrm{mm}$ axial reconstructed section thickness with a soft-tissue algorithm. Real-time automatic tube current modulation software (CARE Dose 4D; Siemens) was used to regulate the tube current, depending on the patient's anatomy. Images were obtained from the upper margin of the frontal sinus to the top of the aortic arch, either with or without an IV injection of contrast media. Contrast-enhanced CT scans were acquired after 70 seconds following the administration of $150 \mathrm{~mL}$ of intravenous ioversol (Optiray-320; Mallinckrodt, St. Louis, Missouri) at a rate of $2.5 \mathrm{~mL} / \mathrm{s}$ via the right antecubital vein. Radiologic images were reviewed with a PACS.

One neuroradiologist with 7 years of dedicated head and neck experience assessed the image noise by calculating the SD of the CT values in Hounsfield units for pixels within an ROI fitted within the relevant structures (as outlined below). CT values were measured at the mid- and lower neck levels contralateral to the side used for the intravenous contrast media administration. The ROIs were placed on the internal jugular vein and sternocleidomastoid muscle at the level of the cricoid cartilage (midneck) and the thyroid gland and internal jugular vein at the level of the first costovertebral joint (lower neck).

For the evaluation of streak artifacts, 2 board-certified neuroradiologists with 15 and 8 years of experience independently analyzed the axial CT images on a PACS screen, with the scanning parameters removed from the screen to blind the reviewers to whether the arm traction device was being used. All image assessment was performed with a standard soft-tissue setting (window width: 300/window level: 35). The degree of streak artifacts in the supraclavicular fossa was evaluated and scored as follows: 1 , no or minimal artifacts with no image obscuration; 2, mild artifacts causing partial obscuration of subcutaneous fat or skin without diagnostic interference; and 3, severe artifacts causing obscuration of deep cervical structures with diagnostic interference. ${ }^{9}$ The supraclavicular fossa was defined on each axial scan when any portion of the clavicle was identified on 1 side of the neck. ${ }^{12}$

Radiation exposure was evaluated with DLP and CTDI ${ }_{\mathrm{vol}}$. Potential confounds, including the effective diameter of the neck and the $\mathrm{z}$-axis scan range, were also assessed. The effective diameter of the neck was calculated from an axial CT image at the level of the lower margin of the cricoid cartilage using the following equation: Effective Diameter $=\sqrt{ }$ Anteroposterior Diameter $\times$ 

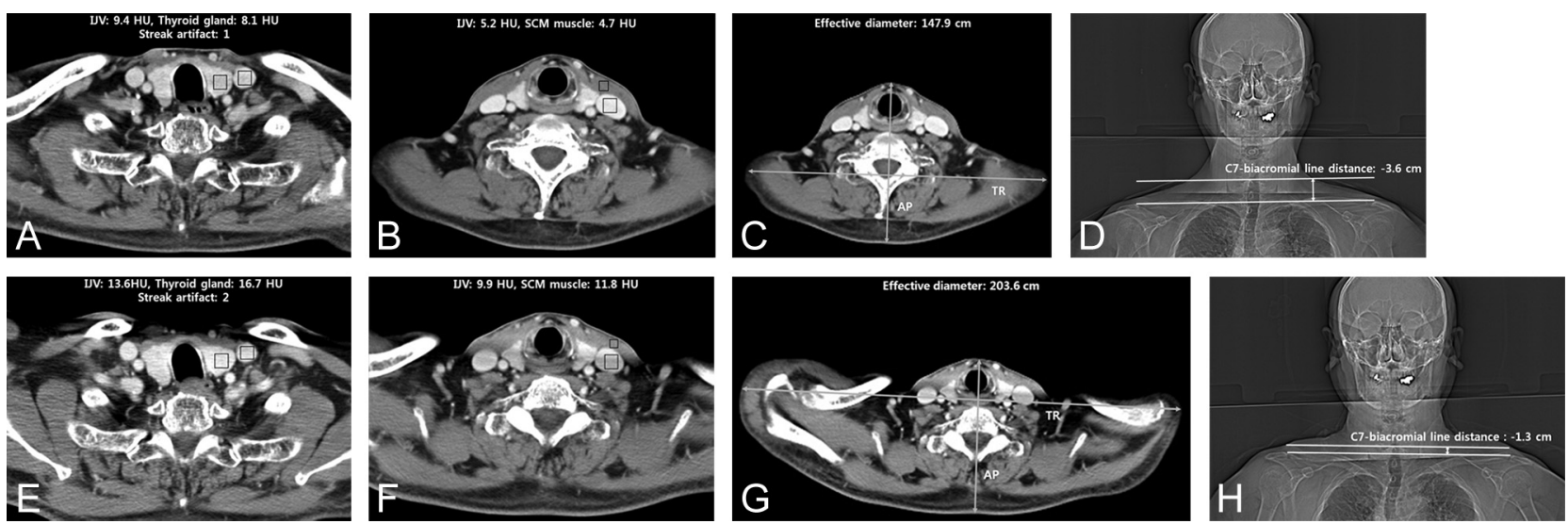

FIG 2. Representative neck $C T$ images obtained with the intervention $(A-D)$ and standard $(E-H)$ protocols. The images demonstrate the measurements for image noise at the lower neck (first costovertebral joint level, $A$ and $E$ ) and midneck (cricoid cartilage level, $B$ and $F$ ), and the effective diameter measurement at the midneck ( $C$ and $G$ ). The $C 7$-biacromial line distance was determined as illustrated on images $D$ and $H$. The white line indicates the biacromial line; arrow, the C7-biacromial line distance; SCM, sternocleidomastoid; IJV, internal jugular vein; HU, Hounsfield units.

Transverse Diameter. ${ }^{13}$ The total scan length was assessed for each patient by multiplying the total number of axial images acquired by the section thickness. To measure the degree of shoulder-level shift, we determined the C7-biacromial line distance. Two straight lines were drawn on the anteroposterior scout image: One was the line connecting the upper margin of the shoulder acromion bilaterally (biacromial line) ${ }^{14}$ while the other line was parallel to the middle of the upper endplate of C7. The C7-biacromial line distance was defined as the distance between the 2 straight lines (Fig 2). The measurement was expressed as negative if the biacromial line was located caudal to the upper margin of $\mathrm{C} 7$ and positive if the line was located cranial to the upper margin of $\mathrm{C} 7$.

After the CT scans with the intervention protocol, the patients were asked to fill in a questionnaire and rate the discomfort on a $10-\mathrm{cm}$ visual analog scale (grades $0-10$ ), with 0 representing "no discomfort" and 10 representing "the worst discomfort imaginable." Patients were also asked if they would be willing to accept the arm traction device at the next CT follow-up.

\section{Statistical Analysis}

A sample size of 59 would achieve $80 \%$ power to detect a mean of paired differences of -9.0 with an estimated SD of differences of 20.0 and a significance level $(\alpha)$ of .0125 using a 2 -sided paired $t$ test (ie, the paired difference of DLP between the standard and intervention protocols). This sample size would achieve $89 \%$ power to detect a mean of paired differences of -3.5 with an estimated SD of differences of 7.0 and a significance level $(\alpha)$ of .0125 using a 2-sided paired $t$ test (ie, the paired difference between the standard and intervention protocols of the image noise on the thyroid gland at the position of the lower neck). Therefore, with consideration of a 15\% follow-up loss, we planned to collect data from 70 patients to estimate the double primary end points (DLP and image noise on the thyroid gland at the lower neck) in each subject from application of the intervention and standard CT protocols.

Data for continuous variables were presented as means and SDs, and for categoric variables, as the number of subjects. Paired $t$ tests were used to analyze the differences between the 2 protocols with respect to image noise, DLP, CTDI ${ }_{\mathrm{vol}}$, effective diameter, C7-biacromial line distance, and $\mathrm{z}$-axis scan range. A marginal

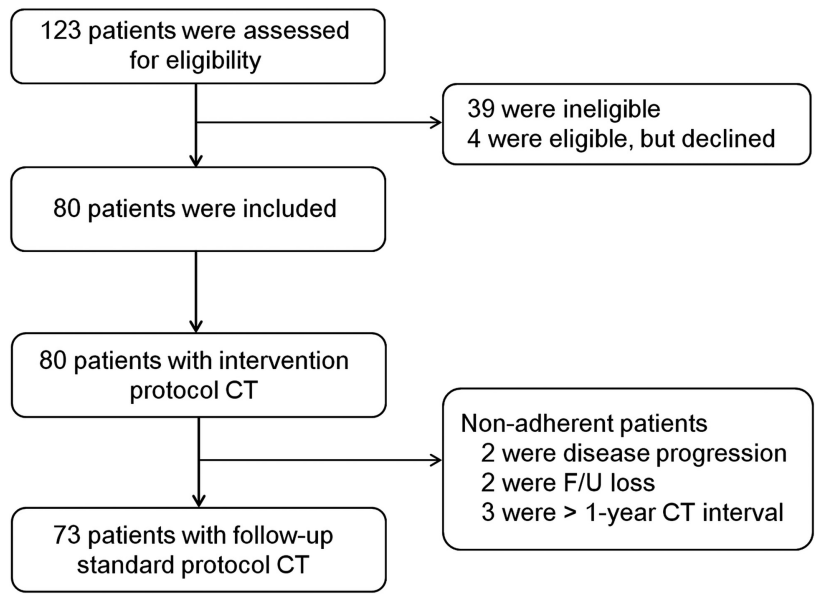

FIG 3. Study flow diagram.

homogeneity test and visual grade characteristics method were used to compare the streak artifact levels between the 2 protocols. The interobserver agreement for subjective evaluation of streak artifacts was calculated with $\kappa$ statistics, and estimation of the overall $\kappa$ was as follows: slight agreement $(0-0.20)$, fair agreement $(0.21-0.40)$, moderate agreement $(0.41-0.60)$, substantial agreement (0.61-0.80), and almost perfect agreement (0.811.00). All statistical analyses were performed with MedCalc for Windows, Version 15.0 (MedCalc Software, Mariakerke, Belgium) and SPSS, Version 23.0 for Windows (SPSS, Armonk, New York), with a $P$ value of .05 considered statistically significant.

\section{RESULTS}

From March 2015 to April 2016, we screened 123 patients, of whom 84 were eligible. After we excluded 4 patients who declined to use the arm traction device, 80 patients were included and underwent CT with the intervention protocol. Seven of these patients $(8.8 \%)$ who were nonadherent to the study protocol for measurement of study outcomes were excluded. Finally, 73 patients (mean age, 50.6 years; age range, 19-76 years) were enrolled and underwent consecutive CT scans with the standard protocol (Fig 3). The baseline characteristics of the patients enrolled in this 
study are summarized in Table 1 . Among 73 patients, 4 patients (5.5\%) underwent nonenhanced CT, with a mean interval between the intervention and standard CT protocol of 155 days.

\section{Primary and Secondary Outcomes}

Image-quality measures based on the assessment of image noise showed that the intervention protocol resulted in statistically significant improvement in CT image quality in the lower neck in comparison with the standard protocol $(P<.001)$. A mean noise reduction of $28.5 \%$ (from 17.2 to $12.3 \mathrm{HU}$; group mean $\mathrm{SD}$ of the values within the ROI) was measured at the thyroid gland and $25.2 \%$ (from 14.3 to $10.7 \mathrm{HU}$ ) at the internal jugular vein in the lower neck. A statistically significant decrease in the DLP was also measured in the intervention protocol compared with the standard protocol $(P<.001$, Table 2$)$.

The evaluation of the streak artifacts in the supraclavicular fossa showed a significant decrease in the intervention protocol in comparison with the standard protocol (a mean score of 1.6 versus 1.9; test of marginal homogeneity, $P<.001)$. The area under the visual grade characteristics curve was determined as $0.61(95 \%$

\begin{tabular}{|c|c|}
\hline Parameter & Value \\
\hline \multicolumn{2}{|l|}{ Age (yr) } \\
\hline Mean & 50.6 \\
\hline Range & 19-76 \\
\hline \multicolumn{2}{|l|}{ Sex (No.) (\%) } \\
\hline Men & $39(53.4)$ \\
\hline Women & $34(46.6)$ \\
\hline \multicolumn{2}{|l|}{ Body weight $(\mathrm{kg})$} \\
\hline Mean & 64.7 \\
\hline Range & $35.7-98.1$ \\
\hline \multicolumn{2}{|c|}{ Interval of CT scans (days) } \\
\hline Mean & 155 \\
\hline Range & $58-355$ \\
\hline
\end{tabular}

Table 2: Clinical outcomes in the intervention and standard CT protocols ${ }^{\mathrm{a}}$

\begin{tabular}{|c|c|c|c|}
\hline Outcome & $\begin{array}{l}\text { Intervention CT } \\
\text { Protocol }(n=73)\end{array}$ & $\begin{array}{c}\text { Standard CT } \\
\text { Protocol }(n=73)\end{array}$ & $P$ Value \\
\hline \multicolumn{4}{|l|}{$\begin{array}{l}\text { Primary end points } \\
\text { Image noise, lower neck }\end{array}$} \\
\hline Thyroid gland & $12.3 \pm 5.1$ & $17.2 \pm 5.5$ & $<.001$ \\
\hline Internal jugular vein & $10.7 \pm 4.4$ & $14.3 \pm 5.1$ & $<.001$ \\
\hline $\mathrm{DLP}(\mathrm{mG} \times \times \mathrm{cm})$ & $397.6 \pm 52.8$ & $413.9 \pm 56.3$ & $<.001$ \\
\hline \multicolumn{4}{|l|}{ Secondary end points } \\
\hline Streak artifacts ${ }^{c}$ & $1.6 \pm 0.5$ & $1.9 \pm 0.4$ & $<.001$ \\
\hline 1 & $26(35.6 \%)$ & $11(15.1 \%)$ & \\
\hline 2 & $46(63.0 \%)$ & $59(80.8 \%)$ & \\
\hline 3 & $1(1.4 \%)$ & $3(4.1 \%)$ & \\
\hline \multicolumn{4}{|l|}{ Image noise, midneck ${ }^{b}$} \\
\hline Internal jugular vein & $7.1 \pm 3.7$ & $9.1 \pm 5.8$ & .009 \\
\hline SCM muscle & $5.6 \pm 2.4$ & $7.3 \pm 4.0$ & $<.001$ \\
\hline $\mathrm{CTDI}_{\mathrm{vol}}(\mathrm{mGy})$ & $13.4 \pm 0.9$ & $13.9 \pm 0.9$ & $<.001$ \\
\hline Effective diameter $(\mathrm{cm})^{\mathrm{d}}$ & $16.2 \pm 4.4$ & $19.6 \pm 4.9$ & $<.001$ \\
\hline C7-biacromial line distance $(\mathrm{cm})^{\mathrm{e}}$ & $-2.4 \pm 1.1$ & $-0.3 \pm 1.1$ & $<.001$ \\
\hline
\end{tabular}

Note:-SCM indicates sternocleidomastoid.

${ }^{a}$ Data are expressed as mean $\pm \mathrm{SD}$.

${ }^{b}$ Based on the evaluation of image noise, by measurement of the SD of the CT values in Hounsfield units.

c Streak artifacts at the supraclavicular fossa (1, none or minimal; 2, mild; and 3, severe).

${ }^{d}$ Effective diameter of the neck at the level of the lower margin of the cricoid cartilage.

e The C7-biacromial line distance was defined as the distance from the intersection of a line connecting the acromion processes to the upper endplate of C7. Negative values indicate that the biacromial line was located caudal to the upper margin of C7, and positive values, that it was located cranial of the upper margin of C7. confidence interval, 0.519-0.702) and was statistically significant $(P=.02)$. The interobserver agreement for streak artifacts was substantial $(\kappa$ value $=0.66 ; 95 \%$ confidence interval, $0.537-$ 0.794). CTDI $_{\mathrm{vol}}$ and effective diameter were also significantly lower in the intervention protocol than in the standard protocol (for both, $P<.001$; Table 2). The intervention protocol could lower the biacromial line by an average of $2.1 \mathrm{~cm}$ in comparison with the standard protocol $(P<.001)$. There was no statistical difference in the $\mathrm{z}$-axis scan range between the 2 protocols (intervention versus standard, $27.2 \pm 3.1 \mathrm{~cm}$ versus $26.8 \pm 2.7 \mathrm{~cm}$; $P=$ .14; Table 2). A representative case is illustrated in Fig 2.

\section{Questionnaire on Intervention Protocol}

All 80 patients filled out the questionnaire given after the neck CT that used the intervention protocol. The mean score on the visual analog scale was 0.6 (range, $0-8)$. Sixty-two patients $(77.5 \%)$ showed no discomfort (score 0 ), while 3 patients $(3.8 \%)$ rated discomfort as $>3$. Seventy-seven patients $(96.2 \%)$ responded that they would be willing to accept the intervention CT protocol again.

\section{DISCUSSION}

We prospectively assessed the effect of an arm traction device combined with automatic tube current modulation on the image quality and radiation exposure level of neck CT. Application of the arm traction device lowered the shoulder level and reduced both the dimension of the volume of tissue of imaging interest and the radiation attenuation from the shoulder and upper thorax. As a result, we could reduce not only the image noise and streak artifacts in the mid- and lower neck but also the radiation exposure level. Our current results suggest that the use of an arm traction device in routine practice could easily improve neck CT image quality while, at the same time, reducing radiation exposure.

In the present study, the main eligibility criterion was patients with lymphoma with complete remission who were scheduled to undergo consecutive neck CT scans within a 1-year interval. The National Comprehensive Cancer Network guidelines for patients with lymphoma recommend surveillance CT scans every 6 or 12 months for 2 years after completion of treatment. ${ }^{15}$ We therefore considered these patients ideal for our study design-that is, the same patients would be eligible for both intervention and standard CT protocols. Even though the main eligibility criterion in this study was patients with lymphoma with complete remission, the arm traction device could be applied to any patient who would be cooperative in routine practice because this device is easy to use and causes little discomfort for most patients.

Previous studies have investigated several techniques to prevent image degradation in the lower neck and supracla- 
vicular fossa. Some of the studies suggested the potential utility of lowering the shoulder level to reduce image degradation. However, the subjects or CT machines used in the studies demonstrated considerable heterogeneity between the control and intervention groups, ${ }^{8-10}$ which may have greatly influenced the study results. We therefore designed a prospective study that used the same CT scanner for all acquisitions and enrolled the identical study subjects in both the intervention and control groups, to test the exact effect of the arm traction device on CT image quality and radiation exposure. This study revealed that the arm traction device we designed lowered the biacromial line an average of $2.1 \mathrm{~cm}$, which lessened image degradation from the bony shoulder girdles and the abrupt size increase from the circular neck to the broadened chest in the mid- and lower neck scan sections. Given that the automatic tube current modulation technique allows maintenance of constant image quality within a maximum radiation exposure level by rapidly responding to large variations in beam attenuation, reducing the dimensions of the tissue of interest to be scanned (ie, effective diameter) should be a basic step toward the reduction of radiation exposure, as demonstrated in this study.

This study has several limitations. First, our subjects underwent imaging with a single CT machine to reduce any influence on the results related to the intrinsic properties of different CT systems. To generalize the study results, further studies on different CT machines are required, even though we speculate that future study results will be similar to ours, because the basic principle is similar across CT machines. Second, we did not consider the possibility of thyroiditis in the measurement of the ROIs. Any chronologic changes in thyroiditis could have influenced the image noise in the thyroid gland. However, we believe that any effects from thyroiditis must be extremely small because the image noise of both the thyroid gland and the internal jugular vein in the lower neck showed similar tendencies (reductions of 25.2\%$28.5 \%$ in the intervention protocol). Third, we could not assess the size-specific dose estimate, which is the estimate of the absorbed radiation dose in an individual patient. A recent recommendation for studies evaluating the CT dose level suggested that the following 4 parameters be assessed to provide an accurate estimation of the dose absorbed by an individual patient: 1) $\mathrm{CTDI}_{\mathrm{vol}}$, 2) DLP, 3) effective diameter, and 4) size-specific dose estimates. ${ }^{13}$ However, we did not report the size-specific dose estimates in the present investigation because conversion factors for this calculation are currently available for only the torso (chest-abdomen and/or pelvis), not for the neck region. ${ }^{16,17} \mathrm{Fi}-$ nally, the clinical applicability of the arm traction device is limited to cooperative patients with no physical disability in either arm.

\section{CONCLUSIONS}

An arm traction device improves the image quality and reduces radiation exposure during neck CT. This arm traction device can be simply applied in cooperative patients with suspected lower neck lesions, and the approach offers distinct advantages over the conventional imaging protocol.
Disclosures: Jung Hwan Baek—UNRELATED: Consultancy: STARmed, RF Medical Co.

\section{REFERENCES}

1. Kalra MK, Maher MM, Toth TL, et al. Strategies for CT radiation dose optimization. Radiology 2004;230:619-28 CrossRef Medline

2. Russell M, Fink J, Rebeles F, et al. Balancing radiation dose and image quality: clinical applications of neck volume CT. AJNR Am J Neuroradiol 2008;29:727-31 CrossRef Medline

3. Tamm EP, Rong XJ, Cody DD, et al. Quality initiatives: CT radiation dose reduction: how to implement change without sacrificing diagnostic quality. Radiographics 2011;31:1823-32 CrossRef Medline

4. Parakh A, Kortesniemi M, Schindera ST. CT radiation dose management: a comprehensive optimization process for improving patient safety. Radiology 2016;280:663-73 CrossRef Medline

5. Goenka AH, Herts BR, Dong F, et al. Image noise, CNR, and detectability of low-contrast, low-attenuation liver lesions in a phantom: effects of radiation exposure, phantom size, integrated circuit detector, and iterative reconstruction. Radiology 2016;280:475-82 CrossRef Medline

6. Kim JH, Choo KS, Moon TY, et al. Comparison of the image qualities of filtered back-projection, adaptive statistical iterative reconstruction, and model-based iterative reconstruction for CT venography at 80 kVp. Eur Radiol 2016;26:2055-63 CrossRef Medline

7. Yoon HJ, Chung MJ, Hwang HS, et al. Adaptive statistical iterative reconstruction-applied ultra-low-dose CT with radiography-comparable radiation dose: usefulness for lung nodule detection. $\mathrm{Ko}$ rean J Radiol 2015;16:1132-41 CrossRef Medline

8. Wirth S, Meindl T, Treitl M, et al. Comparison of different patient positioning strategies to minimize shoulder girdle artifacts in head and neck CT. Eur Radiol 2006;16:1757-62 CrossRef Medline

9. Choi YJ, Lee JH, Cho WY, et al. Effect of an arm traction device on image quality and radiation exposure during neck computed tomography. Eur J Radiol 2016;85:68-72 CrossRef Medline

10. Kranz PG, Wylie JD, Hoang JK, et al. Effect of the CT table strap on radiation exposure and image quality during cervical spine CT. AJNR Am J Neuroradiol 2014;35:1870-76 CrossRef Medline

11. Brink M, de Lange F, Oostveen LJ, et al. Arm raising at exposurecontrolled multidetector trauma CT of thoracoabdominal region: higher image quality, lower radiation dose. Radiology 2008;249: 661-70 CrossRef Medline

12. Som PM, Curtin HD, Mancuso AA. Imaging-based nodal classification for evaluation of neck metastatic adenopathy. AJR Am J Roentgenol 2000;174:837-44 CrossRef Medline

13. Bankier AA, Kressel HY. Through the Looking Glass revisited: the need for more meaning and less drama in the reporting of dose and dose reduction in CT. Radiology 2012;265:4-8 CrossRef Medline

14. Korovessis P, Koureas G, Zacharatos S, et al. Backpacks, back pain, sagittal spinal curves and trunk alignment in adolescents: a logistic and multinomial logistic analysis. Spine (Phila Pa 1976) 2005;30: 247-55 CrossRef Medline

15. Zelenetz AD, Gordon LI, Wierda WG, et al. Diffuse Large B-Cell Lymphoma Version 1.2016. J Natl Compr Canc Netw 2016;14:196231 Medline

16. American Association of Physicists in Medicine. Size-specific dose estimates (SSDE) in pediatric and adult body CT examinations. AAPM Report 204. College Park, Maryland: American Association of Physicists in Medicine; 2011

17. Christner JA, Braun NN, Jacobsen MC, et al. Size-specific dose estimates for adult patients at CT of the torso. Radiology 2012;265: 841-47 CrossRef Medline 